\title{
GENERALIZATION OF $\omega$-CLOSED SETS VIA OPERATORS AND IDEALS
}

\author{
C. CARPINTERO, E. ROSAS, M. SALAS, J. SANABRIA AND L. VÁSQUEZ
}

\begin{abstract}
A new type of closed sets in a topological space, called $\omega_{\mathcal{I}, \gamma^{-}}$ closed sets, is introduced and studied. Also we studied and characterized the class of spaces having $(\mathcal{I}, \gamma)$-tightness.
\end{abstract}

\section{INTRODUCTION}

The concept of $\omega$-closed set was introduced by Arhangel'skii [1], as a generalization of the classical concept of closed set in term of countable sets. The collection formed by the complement of the $\omega$-closed sets in a topological space $(X, \tau)$ form a topology on $X$ finer than $\tau$. In the case that the collection of $\omega$-closed sets coincide with the collection of closed sets, it is said that $X$ has countable tightness. The importance of the spaces that have countable tightness is that the closure of any subset can be determined in terms of countable sets. Ekici and Jafari [3], introduced the notion of $\omega_{*}$-closed sets as a generalization of the concept introduced by Arhangel'skii [1] in terms of $\theta$-closure. In this paper, we introduce and study in terms of an operator associated with a topology [5] and using topological ideals [6], the notion of $\omega_{\mathcal{I}, \gamma^{-}}$-losed sets as a unified form of $\omega$-closed sets [1] and $\omega_{*}$-closed sets [3]. Also we study fundamental properties of $\omega_{\mathcal{I}, \gamma}$-closed sets and we determine its closure in terms of topological ideals.

\section{Preliminaries}

Let $(X, \tau)$ be a topological space and $A \subseteq X$, the closure and the interior of $A$ are denoted by $\operatorname{cl}(A)$ and $\operatorname{int}(A)$ respectively. A subset $A$ of $X$ is called $\omega$-closed [1], if $\operatorname{cl}(B) \subset A$ whenever $B \subset A$ and $B$ is a countable set. The complement of an $\omega$-closed set is called $\omega$-open, the collection of all $\omega$-open sets form a topology on $X$ denoted by $\tau_{\omega}[1]$.

2010 Mathematics Subject Classification. 54C10, 54D10.

Key words and phrases. $\omega$-closed sets, operations, ideals. 
If $X$ has countable tightness then the closure of any subset of $X$ can be determined in terms of countable sets, that is, $X$ has countable tightness if and only if for any subset $A$ of $X$, if $x \in \operatorname{cl}(A)$ then there exists a countable set $B$ such that $B \subset A$ and $x \in \operatorname{cl}(B)$. A classical example of spaces having countable tightness are the first countable spaces.

The $\theta$-closure [8] of a subset $A$ of $X$, denoted by $\theta-c l(A)$, is defined as the set of all $x \in X$ such that $\operatorname{cl}(U) \cap A \neq \emptyset$ for all open subset $U$ of $X$ containing $x$. A subset $A$ of $X$ is called $\theta$-closed if $A=\theta-\operatorname{cl}(A)$. The $\theta$-interior of $A$, denoted by $\theta-\operatorname{int}(A)$, is defined as the union of all open subsets $U$ of $X$ such that $c l(U) \subseteq A$.The collection of all $\theta$-open sets form a topology on $X$ denoted by $\tau_{\theta}$. A subset $A$ of $X$ is called $\omega_{*}$-closed [3], if $\theta-c l(B) \subset A$ whenever $B \subset A$ and $B$ is a countable set. The complement of an $\omega_{*}$-closed set is called $\omega_{*}$-open. The collection of all $\omega_{*}$-open sets, denoted by $\tau_{\omega_{*}}$, form a topology on $X$ finer than $\tau$. Every $\omega$-closed set is $\omega_{*}$-closed [3]. Note that the $\omega_{*}$-closed sets are the $\omega$-closed sets in the space $\left(X, \tau_{\theta}\right)$.

Definition 2.1. ([5], [7]) Let $(X, \tau)$ be a topological space. An application $\gamma: 2^{X} \rightarrow 2^{X}$ is said to be an operator associated to $\tau$, if $U \subset \gamma(U)$ for each $U \in \tau$.

An operator $\gamma$ is said to be regular [5], if for every pair of open sets $U$, $V$ such that $x \in U \cap V$ there is an open set $W$ such that $x \in W$ and $\gamma(W) \subset \gamma(U) \cap \gamma(V)$.

Definition 2.2. ([4]) Let $(X, \tau)$ be a topological space, and $\gamma$ an operator associated to $\tau$. For a subset $A$ of $X$, the $\gamma$-closure of A and the $\gamma$-interior of $\mathrm{A}$ are defined as:

(1) $\operatorname{cl}_{\gamma}(A)=\{x \in X: \gamma(U) \cap A \neq \emptyset$ for all $U \in \tau, x \in U\}$

(2) $\operatorname{int}_{\gamma}(A)=\{x \in A: x \in U \in \tau$ and $\gamma(U) \subset A\}$

A subset $A$ of $X$ is said to be $\gamma$-open [4] if $A=i n t_{\gamma}(A)$. The complement of a $\gamma$-open set is said to be $\gamma$-closed. All $\gamma$-closed set is a closed set.

Remark 2.3. Some classical closed sets can be obtained from the Definition 2.2 choosing specific operators, as follows:

(1) If $\gamma=i d$ then $c l_{\gamma}(A)=\operatorname{cl}(A), \operatorname{int}_{\gamma}(A)=\operatorname{int}(A)$ and the $\gamma$-open sets coincide with the open sets.

(2) If $\gamma=c l$ then $c l_{\gamma}(A)=\theta-c l(A), \operatorname{int}_{\gamma}(A)=\theta$-int $(A)$ and the $\gamma$-open sets coincide with the $\theta$-open sets [8].

(3) If $\gamma=$ intcl then $c l_{\gamma}(A)=\delta$-cl $(A)$, int ${ }_{\gamma}(A)=\delta$-int $(A)$ and the $\gamma$-open sets coincide with the $\delta$-open sets [8].

Theorem 2.4. Let $(X, \tau)$ be a topological space, and $\gamma$ an operator associated to $\tau$. For any $A$ and $B$ subsets of $X$, we have the following relations: 
(1) $A \subset \operatorname{cl}(A) \subset c l_{\gamma}(A)$.

(2) If $A \subset B$ then $\operatorname{cl}_{\gamma}(A) \subset \operatorname{cl}_{\gamma}(B)$.

(3) $\operatorname{cl}_{\gamma}(A) \cup \operatorname{cl}_{\gamma}(B) \subset \operatorname{cl}_{\gamma}(A \cup B)$,

(4) $X \backslash \operatorname{cl}_{\gamma}(A)=\operatorname{int}_{\gamma}(X \backslash A)$.

(5) $A$ is a $\gamma$-closed set if and only if $A=\operatorname{cl}_{\gamma}(A)$.

Note that equality in item (3) of the previous theorem is obtained if $\gamma$ is a regular operator.

Definition 2.5. ([6]) An ideal $\mathcal{I}$ on a nonempty set $X$ is a collection of subsets of $X$ which satisfies:

(1) $A_{1} \in \mathcal{I}$ and $A_{2} \in \mathcal{I}$ implies $A_{1} \cup A_{2} \in \mathcal{I}$

(2) $A_{1} \in \mathcal{I}$ and $A_{2} \subset A_{1}$ implies $A_{2} \in \mathcal{I}$

Throughout this paper by a topological ideal space $(X, \tau, \mathcal{I})$ we will always mean a topological space $(X, \tau)$ together an ideal $\mathcal{I}$ on $X$.

Example 2.6. The following important ideals in a space $(X, \tau)$ will be denoted as follows:

(1) $\mathcal{F}$ the ideal of finite subsets of $X$.

(2) $\mathcal{C}$ the ideal of countable subsets of $X$.

(3) $\mathcal{N}$ the ideal of nowhere dense sets in $(X, \tau)$.

(4) $\mathcal{M}$ the ideal of meager sets in $(X, \tau)$.

(5) $\mathcal{K}$ the ideal of relatively compact sets in $(X, \tau)$.

(6) $\mathcal{C D}$ the ideal of closed discrete sets in $(X, \tau)$.

Definition 2.7. An ideal $\mathcal{I}$ on a space $(X, \tau)$ satisfy the property $(*)$ if $\{x\} \in \mathcal{I}$ for all $x \in X$.

Note that the ideals $\mathcal{F}, \mathcal{C}, 2^{X}, \mathcal{C D}$ satisfies the property $(*)$. If $X$ is a $T_{1}$ space such that the discrete topology is strictly more finer than $\tau$ then the ideals $\mathcal{N}, \mathcal{M}, \mathcal{K}$ satisfies the property $(*)$.

Theorem 2.8. Let $(X, \tau)$ be a space with an ideal $\mathcal{I}$ on $X$. Then the following collection

is a topology on $X$.

$$
\tau_{\mathcal{I}}=\{U \subset X: X \backslash U \in \mathcal{I}\} \cup\{\emptyset\}
$$

Proof. From the definition of $\tau_{\mathcal{I}}$, it follows that $\emptyset \in \tau_{\mathcal{I}}$ and since $\emptyset \in \mathcal{I}$ for any ideal $\mathcal{I}$, then $X \in \tau_{\mathcal{I}}$.

Let $\left\{U_{\alpha}\right\}_{\alpha \in J}$ be an arbitrary collection of elements of $\tau_{\mathcal{I}}$, assume that $U_{\alpha_{0}} \neq \emptyset$ for some $\alpha_{0} \in J$. Note that

$$
X \backslash\left(\cup_{\alpha \in J} U_{\alpha}\right)=\cap_{\alpha \in J}\left(X \backslash U_{\alpha}\right) \subset X \backslash U_{\alpha_{0}} .
$$

As $U_{\alpha_{0}} \in \tau_{\mathcal{I}}$ then $X \backslash U_{\alpha_{0}} \in \mathcal{I}$. Thus $X \backslash\left(\cup_{\alpha \in J} U_{\alpha}\right) \in \mathcal{I}$ and therefore $\cup_{\alpha \in J} U_{\alpha} \in \tau_{\mathcal{I}}$. 
Let $U_{1}$ and $U_{2}$ two elements of $\tau_{\mathcal{I}}$, assume that $U_{1} \neq \emptyset$ and $U_{2} \neq \emptyset$, then $X \backslash U_{1} \in \mathcal{I}$ and $X \backslash U_{2} \in \mathcal{I}$. But,

$$
\left(X \backslash U_{1}\right) \cup\left(X \backslash U_{2}\right) \in \mathcal{I} .
$$

Hence $X \backslash\left(U_{1} \cap U_{2}\right) \in \mathcal{I}$ here we conclude that $U_{1} \cap U_{2} \in \tau_{\mathcal{I}}$.

Note that an ideal $\mathcal{I}$ satisfies the property $(*)$ if only if $\left(X, \tau_{\mathcal{I}}\right)$ is a $T_{1}$ space.

If $(X, \tau)$ is a topological space then the topologies $\tau_{\mathcal{F}}$ and $\tau_{\mathcal{C}}$ coincide with the finite complement and countable complement topologies on $X$.

\section{3. $\omega_{\mathcal{I}, \gamma^{-C L O S E D ~ S E T S ~}}$}

In this section we define a new class of sets, using operators and ideals, which generalizes the $\omega$-closed, $\omega_{*}$-closed, $\theta$-closed and the $\gamma$-closed sets introduced in [1], [3], [8] and [4] respectively. Also we find some additional properties.

Definition 3.1. Let $(X, \tau, \mathcal{I})$ be a topological ideal space and $\gamma$ an operator associated to $\tau$. We say that a subset $A \subset X$ is $\omega_{\mathcal{I}, \gamma}$-closed set if

$$
\operatorname{cl}_{\gamma}(B) \subset A \text { for all } B \subset A \text {, such that } B \in \mathcal{I} \text {. }
$$

The complement of an $\omega_{\mathcal{I}, \gamma^{-}}$-losed set is called $\omega_{\mathcal{I}, \gamma^{-}}$-open.

Remark 3.2. It is clear that

(1) Every $\gamma$-closed set is an $\omega_{\mathcal{I}, \gamma}$-closed set.

(2) Every $\omega_{\mathcal{C}, \gamma}$-closed set is an $\omega$-closed set.

The following examples show that the concepts of closed set and $\omega_{\mathcal{I}, \gamma^{-}}$ closed set are independent.

Example 3.3. Let $X$ be a nonempty set endowed with the finite complement topology, $\gamma$ the operator $c l$ and $\mathcal{I}$ any ideal. The only $\omega_{\mathcal{I}, \gamma}$-closed sets are $\emptyset$ and $X$.

Example 3.4. Let $(X, \tau)$ be topological space, $\gamma$ the operator $i d$ and $\mathcal{I}$ any ideal. Note that a subset $A$ of $X$ is closed in $\tau_{\mathcal{I}}$ if and only if $A \in \mathcal{I}$ or $A$ is $X$. However, for all $A \subset X$ we have that $A$ is an $\omega_{\mathcal{I}, i d}$-closed set in $\left(X, \tau_{\mathcal{I}}\right)$.

The following example shows the existence of an $\omega_{\mathcal{I}, \gamma}$-closed set that is not a $\gamma$-closed set.

Example 3.5. Consider $\mathbb{R}$ the set of the real numbers with the usual topology and the ideal $\mathcal{C}$ on $\mathbb{R}$. $(0,1)$ is not open in $\left(\mathbb{R}, \tau_{\mathcal{C}}\right)$ but it is an $\omega_{\mathcal{C}, i d}$-open.

The next example shows the existence of an $\omega$-closed set that is not an $\omega_{\mathcal{C}, \gamma^{-}}$closed set. 
Example 3.6. Consider $X$ nonempty set with the topology complement countable, $\gamma$ the operator $c l$ and the ideal $\mathcal{C}$ on $X$. For all $A \subset X$ we have that $A$ is $\omega$-closed. However, if $A$ is a nonempty proper subset of $X$ then $A$ is not an $\omega_{\mathcal{C}, \gamma}$-closed set.

Remark 3.7. Some classical closed sets can be obtained from Definition 3.1 choosing specific operators and ideals, as follows:

(1) If $\gamma=i d$ and $\mathcal{I}=\mathcal{C}$ then the $\omega_{\mathcal{I}, \gamma}$-closed sets coincide with the $\omega$-closed sets.

(2) If $\gamma=c l$ and $\mathcal{I}=\mathcal{C}$ then the $\omega_{\mathcal{I}, \gamma^{-}}$closed sets coincide with the $\omega_{*}$-closed sets.

(3) If $\gamma=i d$ and $\mathcal{I}=2^{X}$ then the $\omega_{\mathcal{I}, \gamma^{-c l o s e d}}$ sets coincide with the closed sets.

(4) If $\gamma=c l$ and $\mathcal{I}=2^{X}$ then the $\omega_{\mathcal{I}, \gamma^{-}}$closed sets coincide with the $\theta$-closed sets.

(5) If $\gamma=$ intcl and $\mathcal{I}=2^{X}$ then the $\omega_{\mathcal{I}, \gamma^{-}}$closed sets coincide with the $\delta$-closed sets.

(6) If $\gamma$ any operator and $\mathcal{I}=2^{X}$ then the $\omega_{\mathcal{I}, \gamma^{-c}}$ closed sets coincide with the $\gamma$-closed sets.

(7) If $\gamma$ any operator and $\mathcal{I}=\{\emptyset\}$ then the class of $\omega_{\mathcal{I}, \gamma^{-}}$closed sets coincide with $2^{X}$.

Theorem 3.8. Let $(X, \tau, \mathcal{I})$ be a topological ideal space and $\gamma$ an operator associated to $\tau$. If $\left\{U_{\alpha}: \alpha \in J\right\}$ is a collection of $\omega_{\mathcal{I}, \gamma \text {-open sets, then }}$ $\bigcup_{\alpha \in J} U_{\alpha}$ is an $\omega_{\mathcal{I}, \gamma}$-open set.

Proof. Given $B \in \mathcal{I}$ such that

$$
B \subset X \backslash\left(\cup_{\alpha \in J} U_{\alpha}\right)
$$

then $B \subset X \backslash U_{\alpha}$ for all $\alpha \in J$, so that

$$
c l_{\gamma}(B) \subset X \backslash U_{\alpha} \text { for all } \alpha \in \mathrm{J} .
$$

Therefore

$$
c_{\gamma}(B) \subset \cap_{\alpha \in J}\left(X \backslash U_{\alpha}\right)=X \backslash\left(\cup_{\alpha \in J} U_{\alpha}\right) .
$$

This implies that $X \backslash\left(\cup_{\alpha \in J} U_{\alpha}\right)$ is $\omega_{\mathcal{I}, \gamma}$-closed.

As an immediate consequence of previous theorem and the De Morgan's laws, we obtain the following corollary.

Corollary 3.9. An arbitrary intersection of $\omega_{\mathcal{I}, \gamma^{-}}$closed sets is an $\omega_{\mathcal{I}, \gamma^{-}}$ closed set.

Theorem 3.10. Let $(X, \tau, \mathcal{I})$ be a topological ideal space and $\gamma$ an regular operator associated to $\tau$. If $U_{1}$ and $U_{2}$ are $\omega_{\mathcal{I}, \gamma}$-open sets, then $U_{1} \cap U_{2}$ is an $\omega_{\mathcal{I}, \gamma}$-open set. 
Proof. Let $U_{1}$ and $U_{2}$ two $\omega_{\mathcal{I}, \gamma}$-open sets, we will show that $X \backslash\left(U_{1} \cap U_{2}\right)$ is $\omega_{\mathcal{I}, \gamma}$-closed set. Given $B \in \mathcal{I}$ such that

$$
B \subset X \backslash\left(U_{1} \cap U_{2}\right)=\left(X \backslash U_{1}\right) \cup\left(X \backslash U_{2}\right),
$$

we set $B_{1}=B \cap\left(X \backslash U_{1}\right)$ and $B_{2}=B \cap\left(X \backslash U_{2}\right)$. Note that $B_{1}, B_{2} \in \mathcal{I}$ since $B_{1} \subset B, B_{2} \subset B$ and $B \in \mathcal{I}$.

Furthermore, since $B_{1} \subset X \backslash U_{1}$ and $B_{2} \subset X \backslash U_{2}$, we obtain that

$$
\operatorname{cl}_{\gamma}\left(B_{1}\right) \subset X \backslash U_{1}
$$

and

$$
\operatorname{cl}_{\gamma}\left(B_{2}\right) \subset X \backslash U_{2} .
$$

Hence

$$
\operatorname{cl}_{\gamma}\left(B_{1}\right) \cup \operatorname{cl}_{\gamma}\left(B_{2}\right) \subset\left(X \backslash U_{1}\right) \cup\left(X \backslash U_{2}\right)=X \backslash\left(U_{1} \cap U_{2}\right),
$$

and therefore, using the regularity of $\gamma$, it follows that

$$
c l_{\gamma}(B)=\operatorname{cl}_{\gamma}\left(B_{1} \cup B_{2}\right)=\operatorname{cl}_{\gamma}\left(B_{1}\right) \cup \operatorname{cl}_{\gamma}\left(B_{2}\right) \subset X \backslash\left(U_{1} \cap U_{2}\right) .
$$

This implies that $X \backslash\left(U_{1} \cap U_{2}\right)$ is an $\omega_{\mathcal{I}, \gamma}$-closed set.

Corollary 3.11. If $\gamma$ is a regular operator then the collection of $\omega_{\mathcal{I}, \gamma}$-closed sets form a topology.

The following example shows that if the operator $\gamma$ is not regular then the intersection of two $\omega_{\mathcal{I}, \gamma^{-}}$open sets in general is not an $\omega_{\mathcal{I}, \gamma^{-}}$open set.

Example 3.12. Let $X=\{a, b, c\}$ and $\tau=\{\emptyset, X,\{a\},\{b\},\{a, b\},\{a, c\}\}$, the ideal $2^{X}$ on $X$ and $\gamma: 2^{X} \rightarrow 2^{X}$ the operator associated to $\tau$ defined as follows:

$$
\gamma(A)=\left\{\begin{array}{lll}
c l(A) & \text { if } & b \notin A \\
A & \text { if } & b \in A .
\end{array}\right.
$$

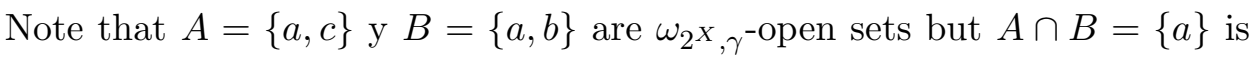
not an $\omega_{2}{ }^{X}, \gamma^{-}$-pen set.

Using the De Morgan's laws in Theorem 3.10, we obtain the following corollary.

Corollary 3.13. If $\gamma$ is a regular operator and $A, B$ are $\omega_{\mathcal{I}, \gamma}$-closed sets then $A \cup B$ is an $\omega_{\mathcal{I}, \gamma}$-closed set.

Theorem 3.14. Let $(X, \tau, \mathcal{I})$ be a topological ideal and $\gamma$ an operator associated to $\tau$, then the following are equivalent:

(1) $A$ is an $\omega_{\mathcal{I}, \gamma^{-}}$open set.

(2) $A \subset \operatorname{int}_{\gamma}(X \backslash B)$ for all $B \in \mathcal{I}$ such that $A \subset X \backslash B$. 
Proof. (1) $\Rightarrow(2)$ Let $B \in \mathcal{I}$ such that $A \subset X \backslash B$, by hypothesis $X \backslash A$ is $\omega_{\mathcal{I}, \gamma}$-closed set, so $c l_{\gamma}(B) \subset X \backslash A$. This implies that

$$
A \subset X \backslash \operatorname{cl}_{\gamma}(B)=\operatorname{int}_{\gamma}(X \backslash B) .
$$

$(2) \Rightarrow(1)$ Let $B \in \mathcal{I}$ such that $B \subset X \backslash A$, by hypothesis $A \subset \operatorname{int}_{\gamma}(X \backslash B)$. Then

$$
X \backslash A \supset X \backslash \operatorname{int}_{\gamma}(X \backslash B) \supset \operatorname{cl}_{\gamma}(B)
$$

As a consequence of the previous theorem, we have.

Corollary 3.15. Let $(X, \tau, \mathcal{I})$ be a topological ideal space and $\gamma$ an operator associated to $\tau$, the following are equivalent:

(1) $A$ is an $\omega_{\mathcal{I}, \gamma}$-open set.

(2) $A \subset \operatorname{int}_{\gamma}(B)$ for all $A \subset B$ such that $X \backslash B \in \mathcal{I}$.

(3) $A \subset \operatorname{int}_{\gamma}(B)$ for all $A \subset B$ such that $B \in \tau_{\mathcal{I}}$.

\section{4. $(\mathcal{I}, \gamma)$-TIGHTNESS SPACE}

The spaces that have countably tightness are essentially those in which the closure of any subset is determined by countable sets. It is proved that every countable space has countable tightness. In this section, we introduce and characterize a new class of spaces that have $(\mathcal{I}, \gamma)$-tightness, which is more general that the spaces having countable tightness due to Arhangel'skii [1]. Also this new class generalize the nowhere dense tightness and the discrete tightness spaces [2].

Definition 4.1. Let $(X, \tau, \mathcal{I})$ be a topological ideal and $\gamma$ an operator associated to $\tau$, we say that $X$ has $(\mathcal{I}, \gamma)$-tightness if every $\omega_{\mathcal{I}, \gamma}$-closed set is a $\gamma$-closed set.

Theorem 4.2. Let $(X, \tau, \mathcal{I})$ be a topological ideal and $\gamma$ an operator associated to $\tau$. Assume that for all $A \subset X$, whenever $x \in c_{\gamma}(A)$ there is $B \in \mathcal{I}$, $B \subset A$ such that $x \in \operatorname{cl}_{\gamma}(B)$, then $X$ has $(\mathcal{I}, \gamma)$-tightness. The converse is true if $\mathcal{I}$ satisfies the property $(*)$.

Proof. Consider $A$ an $\omega_{\mathcal{I}, \gamma}$-closed set and $x \in \operatorname{cl}_{\gamma}(A)$ then by hypothesis there exists $B \in \mathcal{I}$ such that $B \subset A$ and $x \in \operatorname{cl}_{\gamma}(B)$. Since $A$ is an $\omega_{\mathcal{I}, \gamma^{-}}$ closed set, we obtain that $\operatorname{cl}_{\gamma}(B) \subset A$, in consequence, $x \in A$. Therefore $A$ is a $\gamma$-closed set and $X$ has $(\mathcal{I}, \gamma)$-tightness.

For the converse, suppose that $\mathcal{I}$ satisfies the property $(*)$ and that there exists a subset $A$ of $X$ such that $x \in \operatorname{cl}_{\gamma}(A)$. Then for all subset $B \in \mathcal{I}$ such that $B \subset A$, we have $x \notin c l_{\gamma}(B)$.

Observe that $A$ is $\omega_{\mathcal{I}, \gamma}$-closed but is not $\gamma$-closed. In effect, if $B \in \mathcal{I}$ such that $B \subset A$ and $x \notin A$, then we analyze the following cases: 
(i) $x \in c l_{\gamma}(A)$ and $x \notin c l_{\gamma}(B)$.

(ii) $x \notin c l_{\gamma}(A)$.

In the either of the two case, we obtain that $x \notin c l_{\gamma}(B)$, therefore $c l_{\gamma}(B) \subset$ $A$. It follows that $A$ is an $\omega_{\mathcal{I}, \gamma}$-closed set.

Now consider $x \in \operatorname{cl}_{\gamma}(A)$, if $x \in A$ then $\{x\} \in \mathcal{I}$ and $\{x\} \subset A$, Using hypothesis, we conclude that $x \notin \operatorname{cl}_{\gamma}(\{x\})$ which is a contradiction. As a consequence, $A$ is not $\gamma$ closed.

The converse of the previous theorem in general is not true if the ideal does not satisfies the property $(*)$, as is shown in the following example.

Example 4.3. Consider $\mathbb{R}$ the set of the real numbers with the discrete topology, the only nowhere dense set is $\emptyset$. Observe that $\mathbb{R}$ has $(\mathcal{N}, i d)$ tightness and does not satisfies the property $(*)$, also if $A$ is a proper subset of $X$ and $x \in \operatorname{cl}(A)$, then the only element of $\mathcal{N}$ that is contained in $A$ is the empty set, so, $x \notin \operatorname{cl}(\emptyset)$.

Theorem 4.2 says that if

$$
c l_{\gamma}(A)=\bigcup\left\{c l_{\gamma}(B): B \subset A \text { and } B \in \mathcal{I}\right\}
$$

for all $A \subset X$, then $X$ has $(\mathcal{I}, \gamma)$-tightness, and that the converse is true if $\mathcal{I}$ satisfies the property $(*)$.

Remark 4.4. Observe that the notions of countable tightness, nowhere dense tightness and discrete tightness agree with the notion of $(\mathcal{I}, i d)$-tightness when we choose the ideal $\mathcal{I}$. As we can see.

(1) The notion of $(\mathcal{C}, i d)$-tightness coincide with the notion of countable tightness. [1].

(2) The notion of $(\mathcal{N}, i d)$-tightness and $T_{1}$ coincide with the notion of nowhere dense tightness. [2].

(3) The notion of $(\mathcal{C D}, i d)$-tightness coincide with the notion of discrete tightness [2].

\section{REFERENCES}

[1] A. V. Arhangelskii, Bicompacta that satisfy the Suslin condition hereditarily Tightness and free sequences, Dokl. Akad. Nauk SSSR, 199 (1971), 1227-1230.

[2] A. Bella and V. I. Malykhin, Tightness and resolvability, Comment. Math. Univ. Carol., 39 (1) (1998), 177-184.

[3] E. Ekici and S. Jafari, On $\omega_{*}$-closed sets and their topology, Acta Univ. Apulensis, 22 (2010), 175-184.

[4] D. S. Janković, On functions with $\alpha$-closed graphs, Glasnik Mat., 18 (1983), 141-148.

[5] S. Kasahara, Operation-Compact Spaces, Math. Jap., 24 (1979), 97-105.

[6] K. Kuratowski, Topologies I, Warszawa, (1933). 
[7] E. Rosas and J. Vielma, Operator-compact and operator connected spaces, Sci. Math., 1 (2) (1998), 1-6.

[8] N. V. Velićko, H-closed topological spaces, Am. Math. Soc. Transl., 78 (1968), 103-118.

(Received: May 5, 2012)

Departamento de Matemáticas

Universidad de Oriente

Núcleo De Sucre Cumaná

Venezuela

carpintero.carlos@gmail.com

ennisrafael@gmail.com

salasbrown@gmail.com

jesanabri@gmail.com

eligiovm85@gmail.com 